\title{
Aspectos metodológicos de la investigación experimental sobre la adquisición de la lectura
}

\author{
Julio Sánchez Meca, Rafael Rabadán Anta, \\ Agustín Romero Medina \\ Universidad de Murcia
}

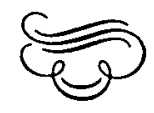

\section{Resumen}

En este trabajo se revisan algunos aspectos de la metodología de la investigación experimental cognitiva. Tras un breve análisis de ciertas cuestiones acerca de los sujetos, aparatos y estímulos, se propone una taxonomia de veinte tareas experimentales, incluyendo procedimientos relacionados con las primeras etapas del procesamiento y otros implicados en las propiedades del procesador central. Estas tareas están especialmente dedicadas a evaluar tres dimensiones cognitivas en el aprendizaje de la lectura: las subbabilidades de los procesos de codificación, el estado de las rutas de acceso al léxico y las propiedades del sistema cognitivo (tales como la velocidad y la precisión de las estrategias de procesamiento).

Palabras clave: Adquisición de la lectura; metodología de la investigaicón en lectura; tareas experimentales cognitivas; retraso específico en lectura.

\section{Abstract}

In this paper some aspects of methodology of cognitive experimental research on reading acquisition are reviewed. After a brief analysis of some subject, aparatus and stimulus critical aspects, a taxonomy of twenty experimental tasks is proposed including procedures related with the earle processing stages, and tasks linked to the properties of central processor. The tasks are specially devoted to evaluate three cognitive dimensions in early reading: subskills of coding processes, the state of lexical access routes, and properties of cognitive system (such as speed and accuracy of processing strategies).

Key words: Reading acquisition; reading research methodology; cognitive experimental tasks; specific reading disability.

Agradecimientos: La presente investigación ha sido subvencionada por una Beca de la Comisión Asesora de Investigación Científica y Técnica, CAICYT, concedida al Dr. Manuel Ato García (ref. PA85-0258).

Dirección de contacto: Dr. Julio Sánchez Meca, Dpto, Metodología y Análisis del Comportamiento, Facultad de Filosofía, Psicología y CC EE, Campus de Espinardo, 30071 Murcia. 


\section{INTRODUCCION}

En los últimos años, el estudio de los procesos cognitivos implicados en el aprendizaje de la lectura, especialmente desde el enfoque del procesamiento de la información, se ha convertido en un área de investigación muy prometedora, tanto por la enorme cantidad de trabajos como por los interesantes logros que se están alcanzando.

Sin embargo, no se ha prestado mucho interés a los complejos aspectos metodológicos que la investigación en este campo conlleva. No en vano, uno de los principales problemas con los que se encuentra cualquier interesado en el tema del aprendizaje de la lectura, tratado desde el enfoque cognitivo actual, reside en una cierta dificultad para entender la metodología empleada en estos estudios. Al no estar familiarizado con las técnicas, tareas y paradigmas experimentales de uso común en este marco de investigación, la comprensión de los resultados obtenidos se hace sumamente ardua y, por decirlo de un modo más gráfico, al tener que fijarse tanto en los árboles apenas si se puede ver el bosque.

No es fácil comprender el modo en que se logra la evidencia empírica que sostiene a los modelos teóricos explicativos de la adquisición lectora. Tampoco es frecuente hallar en la literatura obras o artículos dedicados específicamente a la metodología de la investigación cognitiva sobre lectura, y mucho menos a la investigación sobre la adquisición lectora. Uno de los pocos recursos consiste en echar mano de los manuales y textos generales de psicología cognitiva, pues la investigación sobre la adquisición de la lectura se sirve de gran parte de la metodología experimental cognitiva (al menos en lo referente a procesos perceptivos, atencionales y de memoria). Pero en estos textos las referencias metodológicas suelen ser escasas o muy generales, relativas a los diversos componentes de la lectura.

El presente trabajo tiene como objetivo ofrecer una amplia panorámica de las tareas experimentales que en los últimos años se están aplicando dentro del enfoque cognitivo de estudio del aprendizaje de la lectura y que están -en mayor o menos medida - relacionadas con el funcionamiento de los procesos de memoria exhaustiva de estas tareas; más bien nos hemos centrado en aquellas que mejor pueden acondicionarse al estudio del aprendizaje de la lectura ( $y$, por tanto, en niños de corta edad), y que en sus fases iniciales tiene como protagonista a los procesos de codificación, ya sea visual, fonológica o semántica.

Nuestra revisión comienza con algunos comentarios relativos a las características de los sujetos y de los aparatos y materiales comúnmente utilizados en este tipo de investigación, centrándose después en la descripción de una taxonomía de las tareas experimentales.

\section{CARACTERISTICAS DE LOS SUJETOS}

Uno de los aspectos críticos de la investigación sobre el aprendizaje de la lectura radica en la delimitación de las características de los sujetos que componen la muestra, sobre todo en lo referente a la descripción del nivel de habilidad lectora y sus trastornos. Es ésta una cuestión extremadamente importante, habida cuenta de la pléyade de tipologías existentes en torno al 
fenómeno disléxico. Así pues, será preciso identificar claramente el tipo de problema o retraso lector que se pretende estudiar. Términos tales como "dislexia evolutiva", "dislexia de desarrollo», «dislexia superficial» o "retraso específico en lectura» se combinan y entremezclan a lo largo de la literatura intentando representar un déficit lector más o menos unitario.

Parece que se está llegando a cierto consenso entre los investigadores de los procesos implicados en la adquisición de la lectura, al menos en varios puntos. En primer lugar, en que hay que distinguir entre niños con trastornos muy específicos en la lectura (pero que progresan normalmente en otras áreas escolares) y niños que tienen un déficit de aprendizaje más global, incluido un nivel de inteligencia por debajo de lo normal (Aaron, 1987). En segundo lugar, la selección de los sujetos debe incluir como criterio la ausencia de deprivación ambiental y problemas afectivos, sensoriales, neurológicos o de retraso mental, así como la existencia de una instrucción escolar adecuada; de lo contrario, los problemas de lectura podrían ser tributarios de dichos factores. $Y$ en tercer lugar, debe darse un retraso lector de al menos un año escolar completo, es decir, un niño se considera mal lector cuando su ejecución lectora se corresponde con la de niños de al menos uno o dos cursos inferiores, siendo un tanto variable el criterio seguido por los investigadores (Stanley, 1975). La muestra de sujetos así obtenida podrá calificarse de "retraso específico en lectura»y, según estudios realizados (Duane, 1974), es éste un colectivo que se estima abarca del 5 al 15\% de la población de niños en edad escolar.

Pero más allá de esta denominación del trastorno y de esta caracterización del retraso específico en lectura, el interés de muchas investigaciones se ha focalizado en la cuestión de la homogeneidad versus heterogeneidad de los trastornos de lectura; esto es, como dice Seymour (1986), no ya en las diferencias entre grupos de buenos y malos lectores, sino en distinguir entre diferentes patrones de trastornos lectores. Es una vieja cuestión (Bronner, 1917; cit. por Olson, Kliegl, Davidson y Foltz, 1985) que, sin embargo, sólo empezó a conceptualizarse teóricamente a partir de Johnson y Myklebust (1967) con su propuesta de dos subtipos de dislexia según que el déficit estuviera focalizado en el procesamiento visual o en el auditivo. Parecidas distinciones han sido propuestas posteriormente por Boder (1971, 1973), Ingram, Mason y Blackburn (1970) y Pirozzolo (1979), entre otros. Si bien las investigaciones todavía no han llegado a superar la polémica entre los que defienden una homogeneidad del trastorno, basada en teorías unicausales, o una heterogeneidad, basada en teorías multicausales (cf. Aaron, 1989; Ellis, 1985; Humphreys y Evett, 1985; Olson, Kliegl, Davidson y Foltz, 1985; Stanovich, 1989; para revisión), sin embargo, cada vez va perfilándose mejor una taxonomía inicial que, en términos de Aaron (1989), distingue entre dislexia (trastornos sobre todo en la codificación) e biperlexia (trastornos sobre todo en la comprensión).

En la caracterización de los sujetos deben incluirse también otras especificaciones técnicas. Con respecto a la procedencia de los sujetos, se debe citar: a) La nacionalidad y la lengua de los sujetos; b) el tipo de centro escolar (normal, de educación especial, asistencia a aulas normales, asistencia a reeducación, etc.), y c) el nivel socioeconómico (clase social predominante en el alumnado, zona geográfica en que se encuentra la institución, así como su localización urbana, periférica o rural). Por otra parte, especificarse la edad y el CI 


\section{6}

expresados estadísticamente según media, desviación típica y rango. También es importante el tipo de pruebas de lectura utilizado para determinar el nivel lector. Una incorrecta elección del criterio para distinguir entre buenos y malos lectores puede ser la causa de la no obtención de resultados relevantes en un estudio.

\section{APARATOS Y MATERIALES}

La especificación de los aparatos en una investigación es fundamental y dependerá de los objetivos generales y del tipo de proceso, paradigma experimental o tarea que se esté implementando. Suelen utilizarse en la investigación sobre la lectura desde simples proyectores de diapositivas hasta ordenadores, pasando por taquistoscopios, osciloscopios y audio-cassettes.

En cuanto al material estimular es preciso dedicar especial atención a la naturaleza de los estímulos y al tipo de exposición o de presentación de los mismos. Como tipos de estímulos, se presentan estímulos lingüísticos (letras, dígitos o unidades mayores tales como sílabas, palabras, pseudopalabras, frases, párrafos, etc.) y no lingüísticos, que incluirían al resto de estímulos posibles, y que a su vez pueden poseer contenido semántico para los sujetos (dibujos, símbolos o formas identificables o «etiquetables» con un nombre) o sin contenido semántico (formas sin sentido con las que el sujeto no esté familiarizado o no le suponen algo significativo - manchas, líneas aisladas, puntos, rejillas, trazos abstractos, etc.).

En lo referente al tipo de exposición estimular, hay que distinguir entre exposiciones únicas (cuando el procedimiento experimental consiste en la presentación del estímulo en una sola ocasión) y exposiciones sucesivas (cuando el procedimiento implica la exposición continuada de uno o varios estímulos en estrecha contigüidad especio-temporal).

\section{UNA TAXOMONIA DE TAREAS EXPERIMENTALES}

En la experimentación cognitiva sobre el aprendizaje de la lectura se han utilizado innumerables tareas experimentales, unas extraídas de la psicología experimental cognitiva en general (sobre todo de la investigación sobre procesos perceptivos y de memoria) y otras elaboradas específicamente para estudiar el proceso lector. Así pues, no es nada fácil una clasificación definitiva de las tareas, dado que son múltiples los criterios que se podrían adoptar. Siguiendo a Lesgold (1983; Lesglod, Resnick y Hammond, 1985), Resnick y Weaver (1979) y Stanoovich (1985), pueden distinguirse tres dimensiones cognitivas desde las que se estudian los componentes del procesamiento que intervienen en el aprendizaje de la lectura: (1) Las subbabilidades o procesos de codificación, tanto a nivel visual como fonológico; (2) el estado de las rutas de acceso al léxico, distinguiendo entre las rutas directa e indirecta, y (3) las propiedades del sistema cognitivo, tales como los procesos de detección y reconocimiento de características y de patrones, la capacidad y persistencia de la memoria icónica, la velocidad de transferencia de ésta a la memoria de trabajo, y la capacidad y estrategias de procesamiento de la información del procesador central. 
Las tareas experimentales que a continuación brevemente describimos pertenecen a algunas de estas dimensiones, e incluso a varias simultáneamente, ya que las subhabilidades de codifición se sitúan a un nivel más molar que las propiedades del sistema cognitivo y las rutas de acceso al léxico. En la Tabla I presentamos un cuadro sinóptico en el que se resumen los aspectos del procesamiento cognitivo que básicamente miden cada una de las tareas.

Siguiendo un orden de menor a mayor complejidad o profundidad de procesamiento, tenemos en primer lugar las tareas del umbral de sensibilidad (Tarea 1), cuyo objetivo es examinar la influencia que pueden tener los procesos de detección de características en el aprendizaje de la lectura. La tarea consiste en presentar un estímulo visual simple (por ejemplo, una rejilla de onda cuadrática con frecuencia especial variable) y determinar el umbral de detección del sujeto variando el tiempo de exposición del estímulo mediante algún procedimiento psicofísico (p. ej., Lovegrove, Martin y Slaghuis, 1986; Martin y Lovegrove, 1984).

Una propiedad del procesamiento de la información vinculada a las primeras etapas de análisis de la información visual es la duración de la persistencia visible (Coltheart, 1980), definida como el tipo tras la finalización real del estímulo durante el cual éste continúa siendo visto por el sujeto. Las tareas experimentales que estiman esta duración, y que además se han aplicado al estudio de los problemas de la lectura, son la de persistencia de la forma (Tarea 2) y la de campos sucesivos. La tarea de persistencia de la forma (Haber y Standing, 1969) consiste en la presentación secuencial e intermitente de un estímulo simple (p. ej., una línea, una rejilla de onda cuadrada, una cruz, etc.). Variando el intervalo inter-estímulos (o lo que es lo mismo, la tasa de intermitencia), el sujeto tiene que decir si el estímulo aparece continuamente o si, por el contrario, percibe algún parpadeo. La tarea de campos sucesivos (Tarea 3) implica la presentación de un estímulo descompuesto aleatoriamente en dos partes, de tal forma que ninguna de las partes individuales permite identificar el estímulo completo (Eriksen y Collins, 1967). Las dos mitades del estímulo se presentan brevemente y en estrecha contigüidad temporal, consistiendo la tarea del sujeto en identificar el estímulo completo. Variando, pues, el intervalo de tiempo entre las dos partes del estímulo, es posible determinar la duración de la persistencia visible. Con estos dos procedimientos, numerosos estudios han estimado la duración de la persistencia visible en grupos de niños con diferente nivel de habilidad lectora (p. ej., Badcock y Lovegrove, 1981; Brannan y Williams, 1988; DiLollo, Hanson y McIntyre, 1983; Howell, Smith y Stanley, 1981; Lovegrove, Martin y Slaghuis, 1986; Martin y Lovegrove, 1987).

Otra tareas experimentales que requieren un mayor análisis del estímulo son las de reconocimiento-comparación (Tarea 4), que se centran en los procesos de reconocimiento de caracteres y consisten en la comparación física de letras u otros símbolos (con o sin sentido) presentados brevemente, o bien en el reconocimiento de formas visuales o de letras (p. ej., Bouma y Legein, 1979; Rayner, 1988; Wolford y Fowler, 1984) o de palabras (Foorman y Liberman, 1989).

El estudio de las impliaciones de la capacidad de la memoria icónica en los problemas de lectura se ha llevado a cabo mediante la tarea del informe parcial (Tarea 5; Sperling, 1960; Averbach y Coriell, 1961). Básicamente, consiste en la presentación de un conjunto de estímulos (letras o símbolos sencillos) 
TABLA I

Cuadro sinóptico de los aspectos cognitivos medidos por las tareas experimentales

\begin{tabular}{|c|c|c|c|c|c|c|c|c|c|c|}
\hline \multirow[b]{3}{*}{ TAREAS EXPERIMENTALES } & \multicolumn{6}{|c|}{ Propiedades del sistema cognitivo } & \multirow{2}{*}{\multicolumn{2}{|c|}{$\begin{array}{c}\text { Estado de las } \\
\text { rutas de acceso } \\
\text { al léxico }\end{array}$}} & \multirow{2}{*}{\multicolumn{2}{|c|}{$\begin{array}{c}\text { Subhabilidades } \\
\text { de la } \\
\text { codificación }\end{array}$}} \\
\hline & \multicolumn{4}{|c|}{$\begin{array}{c}\text { En etapas iniciales } \\
\text { del procesamiento de información }\end{array}$} & \multicolumn{2}{|c|}{$\begin{array}{l}\text { Del procesador } \\
\text { central }\end{array}$} & & & & \\
\hline & $1 \mathrm{a}$ & $1 \mathrm{~b}$ & $1 \mathrm{c}$ & $1 d$ & $1 \mathrm{e}$ & 1f & $2 a$ & $2 b$ & $3 a$ & $3 b$ \\
\hline 1. T. de umbral de sensibilidad & $\mathrm{X}$ & & & & & & & & & \\
\hline 2. T. de persistencia de la forma & & & $\mathbf{X}$ & & & & & & & \\
\hline 3. T. de campos sucesivos & & & $\mathrm{X}$ & & & & & & & \\
\hline 4. T. de reconocimiento-comparación & & $\mathrm{X}$ & & & & & & & & \\
\hline 5. T. de informe parcial & & & $\mathrm{X}$ & & & & & & & \\
\hline 6. T. de enmascaramiento visual & & & & $\mathrm{X}$ & & & & & & \\
\hline 7. T. de Reicher & & & & & $\mathrm{X}$ & & & & $\mathbf{X}$ & $\mathbf{X}$ \\
\hline 8. T. de inspección o búsqueda visual & & & & & & $\mathrm{X}$ & & & $\mathbf{X}$ & \\
\hline 9. T. de Sternberg & & & & & & $\mathbf{X}$ & & & & \\
\hline 10. T. de exploración visual de letras & . & & & & & & $\mathbf{X}$ & $\mathbf{X}$ & & \\
\hline 11. $T$. de identificación de anagramas & & & & & $\mathrm{X}$ & $\mathrm{X}$ & & & & \\
\hline 12. T. de Posner & & & & & & & $\mathbf{X}$ & $\mathrm{X}$ & & \\
\hline 13. T. de Brown-Peterson & & & & & & $\mathbf{X}$ & & & & \\
\hline 14. T. de vocalización concurrente & & & & & & $\mathrm{X}$ & & & & \\
\hline 15. T. de recuerdo libre & & & & & $\mathbf{X}$ & $\mathrm{X}$ & & & & \\
\hline 16. T. de amplitud de memoria & & & & & $\mathbf{X}$ & $\mathbf{X}$ & & & & \\
\hline 17. 'T. de denominación de dibujos & & & & & $\mathrm{X}$ & & & & & \\
\hline 18. Tarea de segmentación fonológica & & & & & & & & & & $\mathrm{X}$ \\
\hline 19. Tarea de pronunciación y rima de pal. & & & & & & & & & & $\mathrm{X}$ \\
\hline
\end{tabular}

1a: Detección de características; 1b: Reconocimiento de patrones; 1c: Duración de la memoria icónica; 1d: Transferencia de la memoria icónica a la memoria de trabajo; 1e: Estrategias de procesamiento; 1f: Capacidad de la memoria de trabajo.

2a: Ruta visual directa o automática; $2 \mathrm{~b}$ : Ruta indirecta fonológica.

3a: Codificación visual; 3b: Codificación fonológica. 
durante un breve espacio temporal seguido de una señal visual o auditiva que indica al sujeto el subconjunto de estímulos que debe recordar. Estudios de este tipo relacionados con el aprendizaje de la letura son los de Loubser y Sharratt (1981) y Morrison, Giorrdani y Nagy (1977).

Estrechamente vinculada a la capacidad de la memoria icónica está la tarea de enmascaramiento visual (Tarea 6) y, más concretamente, a la velocidad de transferencia de la información desde la memoria icónica a la memoria de trabajo. Esta tarea consiste en la presentación sucesiva de dos estímulos visuales, el estímulo-objetivo (que el sujeto tiene que identificar) y el estímulo enmascarador que, presentado en estrecha contigüidad espacio-temporal con el anterior, interfiere su procesamiento dificultando así la identificación. El estímulo-objetivo puede estar compuesto de letras, sílabas, palabras e incluso material no verbal. El estímulo enmascarador puede ser desde un simple flash de luz de fuerte intensidad hasta formas sin sentido compuestas por zonas blancas y negras repartidas aleatoriamente (Breitmeyer y Ganz, 1976). Variando el intevalo entre el estímulo-objetivo y la máscara se puede determinar la velocidad de transferencia de la información en grupos de diferente habilidad lectora. Estudios de estas características son los de Arnett y DiLollo (1979), Ellis y Miles (1977), Grosser y Trzeciak (1981), Lovegrove y Brown (1978), Stanley y Hall (1973), Valera y Sánchez Meca (1989), Williams y LeCluyse (en prensa), Williams, LeCluyse y Bologna (en prensa) y Williams, Molinet y LeCluyse (1989).

Un conjunto de tareas vinculado con el efecto de superioridad de la palabra consiste en presentar dos estímulos (habitualmente series de letras), ante los cuales el sujeto ha de emitir una respuesta «igual/diferente». En estas tareas la variable dependiente es el tiempo de reacción (TR), y el efecto de superioridad de la tarea aparece cuando el TR asociado con el procesamiento de palabras es menor que el obtenido con series de letras no relacionadas entre sí. Por ejemplo, Eichelman (1970) comparó las latencias de respuesta cuando los dos estímulos eran palabras y cuando eran series de letras sin sentido; también manipuló el número de letras que componían tanto las series como las palabras, encontrando que resultaba más rápido procesar las palabras que las series de letras sin sentido, y que la diferencia entre los TR aumentaba a medida que crecía el número de letras componentes. Este paradigma hạ sido utilizado en el campo de la lectura recientemente por Bowers, Steffy y Tate (1988), Fredman y Stevenson (1988), Laxon, Coltheart y Keating (1988) y Olson, Wise, Conners, Rack y Filker (1989).

Otro tipo de técnicas frecuentemente utilizadas en contextos de investigación sobre lectura son las tareas de reconocimiento. En estas tareas el efecto de superioridad de la palabra se manifiesta cuando los sujetos son más precisos en la identificación de una letra cuando ésta se encuentra incluida en una palabra que cuando aparece sola o en series de letras ordenadas aleatoriamente.

En los procedimientos de reconocimiento, el sujeto recibe una lista de ítems (sílabas, palabras, dibujos, etc.) que tiene que memorizar, ya que posteriormente se le presentan éstos mezclados con otros ítems nuevos (distractores). La tarea del sujeto es responder ante cada estímulo presentado si es «nuevo» o "viejo». Otra variante incluye una elección múltiple, en la que el sujeto recibe cada ítem "viejo" con varios distractores, debiendo reconocer y señalar el primero. 
La diferencia entre las tareas de reconocimiento y las de recuerdo es obvia, tanto en el planteamiento (los procesos de recuperación son más complejos en las de recuerdo) como en sus resultados. Por ejemplo, en un experimento de Shepard (1967), los sujetos recibían 540 palabras de aprendizaje obteniendo una tasa de aprendizaje del $88 \%$, tasa absolutamente impensable en tareas de recuerdo con esta dimensión estimular.

Se han observado además diferencias cualitativas entre reconocimiento y recuerdo. Así, el recuerdo libre es facilitado por variables tales como la familiaridad y la similitud de los ítems, variables que, en cambio, disminuyen el rendimiento en el paradigma de reconocimiento. En contraste, la teoría clásica de la fuerza del trazo defiende que las diferencias encontradas en los resultados de ambas técnicas se deben únicamente a que el umbral crítico de reconocimiento tiene un valor inferior al de recuerdo libre. Paradigmas más modernos, como la teoría de la detección de señales, postulan que los ítems «nuevos» juegan el papel de «ruido» y los antiguos que vuelven a presentarse actúan como «señal», determinándose en la ejecución unas tasas de aciertos y de falsas alarmas que arrojan índices de discriminabilidad y de decisión (aunque el primero no tiene en este contexto una interpretación perceptiva, sino mnémica).

La tarea de Reicher (Tarea 7) constituye una variante de este paradigma descrito anteriormente, considerando fundamentalmente el efecto facilitador del contexto, ya sea semántico u ortográfico. En esta tarea se presenta brevemente un estímulo visual seguido inmediatamente por una máscara para eliminar la persistencia de la huella perceptiva. Junto con la máscara, se exponen dos alternativas situadas en la posición serial correspondiente a la letra que se trata de identificar. Por ejemplo, se presenta como estímulo la palabra PATA: a continuación, aparece la máscara solicitando la identificación de la cuarta letra de la secuencia estimular, a lo que el sujeto debe responder eligiendo entre la A y la $\mathrm{O}$ (e incluso la E). La aportación básica de Reicher (1969) fue utilizar como alternativas dos letras que, tanto una como la otra, producían una palabra con sentido. Así, en el caso que nos sirve de ejemplo, el conocimiento del contexto no podía subsanar una deficiencia perceptiva, ya que tanto la $\mathrm{A}$ como la $\mathrm{O}$ hacen que la secuencia adquiera sentido.

Este paradigma suele ser utilizado para comparar situaciones en que el estímulo es una palabra, con otras en que es una letra aislada, una pseudopalabra (es decir, una palabra sin sentido, pero ortográficamente legal) o una nopalabra (secuencia de letras sin sentido e ilegibles, violando las reglas de ortografía). Habitualmente, se observa que cuando la letra aparece en el contexto de una palabra es reconocida correctamente con mayor frecuencia que cuando aparece en cualesquiera de las otras situaciones (véase la tarea de Frederiksen).

La interpretación más plausible en este tipo de tarea es, pues, que la superioridad del reconocimiento de una letra cuando aparece enmarcada contextualmente se debe a que la palabra es reconocida como tal por el sujeto, ya que el efecto aparece incluso cuando las posibilidades de adivinación en base al contexto se igualan para las respuestas correctas e incorrectas, evitándose así el sesgo que podría producirse en el proceso de decisión (Tudela, 1981). Tareas de reconocimiento en sus diversas variantes aparecen recientemente aplicadas al estudio del proceso lector en Holligan y Johnston (1988), Juel 


\section{1}

(1988), Rayner (1988), Stelmack, Saxe, Noldy-Cullum, Campbell y Armitage (1988) y Szeszulski y Manis (1987).

Otro grupo de tareas experimentales tiene su origen en la de inspección o búsqueda visual (Tarea 8), originalmente propuesta por Neisser (1963). Básicamente, consiste en presentar al sujeto un estímulo compuesto por varios renglones de unas seis letras cada uno, radicando su tarea en examinar verticalmente la columna hasta encontrar una determinada letra (por ejemplo, la D), que sólo se encuentra en uno de los renglones. La variable dependiente en esta tarea es el tiempo de búsqueda consumido por el sujeto en encontrar la letra. Como es predecible, los sujetos tardan más en encontrar la letra cuando está al final de la lista que cuando está al principio.

En el tiempo de búsqueda, además de la posición de la letra en la columna, incluye también el tipo de rasgos o patrones físicos de las letras que rodean a la letra-objetivo: Cuando son físicamente muy similares a ésta (por ejemplo, la $\mathrm{O}$, la $\mathrm{Q}$ o la $\mathrm{B}$, en el caso de la $\mathrm{D}$ ), el tiempo de búsqueda se incrementa sensiblemente por el fenómeno de enmascaramiento lateral; cuando, por el contrario, las letras circundantes difieren notoriamente en sus rasgos (por ejemplo, la $\mathrm{Z}$, la W o la $\mathrm{T}$ frente a la $\mathrm{D}$ ), este efecto de contraste facilita la búsqueda, disminuyendo las latencias de respuesta.

La tarea admite múltiples variantes. Por ejemplo, Healy $(1976,1980)$ presentaba un texto y pedía al sujeto que tachara todas las letras «t» que aparecieran. Es ésta, pues, una variante que se ve afectada por variables ligeramente diferentes a las anteriores, pero estrechamente relacionadas. Otra variante basada en la búsqueda de palabras puede encontrarse en la revisión de Hall, White y Guthrie (1986), o en el estudio de Vellutino, Scanlon y Tanzman (1988).

Un paradigma experimental con claras analogías con el que nos ocupa es el desarrollo por Sternberg a finales de los 60 para «...estudiar la forma en que la información es recuperada de la memoria cuando el aprendizaje y la retención son esencialmente perfectos» (Sternberg, 1969, p. 423). El procedimiento experimental básico, conocido como tarea de Stemberg (Tarea 9), consistía en presentar a los sujetos una pequeña serie de ítems o «conjunto positivo», cuyo número oscilaba entre uno y seis. Casi inmediatamente se presenta un «ítem de prueba» y el sujeto debía responder si pertenecía o no al conjunto recién memorizado. La variable dependiente es el TR: las tasas de aciertos y errores no son relevantes, ya que el rendimiento es prácticamente perfecto al no sobrecargarse la capacidad de la memoria de trabajo.

Una combinación de las tareas de búsqueda visual de Neisser y de Sternberg se implementó en la investigación de Ato et al. (1984; véase también López Pina, 1984) y más recientemente Hidalgo y Sánchez Meca (1989), para examinar las implicaciones de los procesos de codificación de la información visual en niños con problemas de lectura. Esta tarea, que hemos denominado tarea de exploración visual de letras (Tarea 10), consiste en presentar filas de letras aisladas por la pantalla de un ordenador, siendo la tarea del sujeto hacer avanzar el cursor a través de las filas desde el teclado e ir marcando aquellas letras idénticas a una letra-objetivo previamente especificada. La tarea de exploración visual de letras posibilita la obtención de un índice de velocidad (basado en el TR) y un índice de detectabilidad (basado en la teoría de detección de señales).

Una tarea experimental clásica ampliamente utilizada en psicología cognitiva 
es la tarea de identificación de anagramas de Frederiksen (Tarea 11; 1977, $1978,1980,1981,1982$ ). Dicha tarea implica presentar brevemente un estímulobase seguido a diferentes intervalos de demora por un estímulo enmascador. El estímulo-base puede ser una palabra, una pseudopalabra o un anagrama sin sentido, es decir, lo que al comentar la tarea de Reicher denominados «nopalabra». La tarea del sujeto consiste en evocar el conjunto de letras que componen el estímulo-base, siendo la variable dependiente crítica el porcentaje de letras correctamente reportadas en cada combinación de niveles de tratamiento. Normalmente, se detecta un incremento en la ejecución con la demora del estímulo enmascador, supuestamente debido a un mayor tiempo de procesamiento disponible para el proceso de codificación de la información. La utilidad de esta tarea para el estudio del procesamiento lector puede contrastarse en los trabajos de Ato et el. (1984) y Velandrino (1985).

La tarea de Posner (Tarea 12) es otro paradigma experimental de gran difusión en investigación básica. Su origen se debe al trabajo de Posner y Mitchell (1967), en el que mostraron a los sujetos pares de letras, teniendo éstos que decidir si eran iguales o diferentes. Este procedimiento incorpora como variable independiente básica la manipulación del criterio para determinar la similaridad-disimilaridad de los pares de letras. Así, las letras de cada par pueden ser: (a) física y nominalmente iguales (por ejemplo, AA); (b) físicamente diferentes y nominalmente iguales $(\mathrm{Aa}) ; \mathrm{y}(\mathrm{c})$ física y nominalmente diferentes (por ejemplo, $\mathrm{Ab}$ ).

Los resultados suelen traslucir latencias mayores para las comparaciones nominales que para las físicas. Esta relación ordinal entre niveles de procesåmiento se mantiene con otros tipos de estímulos (números, figuras, etc.).

Una variante del paradigma inicial de Posner y Mitchell es la introducida por Beller (1970), consistente en presentar a los sujetos hileras de letras de longitud diversa. Pudo comprobar que cuando todas las letras de la hilera eran iguales, el TR se mantenía constante independientemente de su longitud, lo que explicó recurriendo a la teoría del procesamiento en paralelo.

Un estudio reciente sobre diferencias en habilidad lectora con una versión modificada de la tarea de Posner implementada en ordenador puede consultarse en Velandrino y Sánchez Meca (1989).

Una variante de esta tarea ha sido diseñada por Hunt et al. (1981) en un intento de relacionar la comprensión verbal con el acceso a la memoria semántica, partiendo de la distinción entre memoria semántica vs. memoria episódica propuesta por Tulving (1972). Los sujetos tenían por decidir: (a) si una palabra y un dibujo pertenecen a la misma categoría semántica; (b) si dos palabras pertenecen a la misma categoría; y (c) si dos palabras escritas con tipografía diferente eran o no iguales. Las correlaciones más altas $(-0.30)$ se obtuvieron con comprensión lectora, no correlacionando con vocabulario. Los autores explican los resultados aludiendo a un hipotético factor de acceso a la memoria semántica.

En la investigación de las diferencias de procesamiento entre buenos y malos lectores, Cirrin (1984) ha adaptado la tarea para su presentación auditiva. Otros estudiosos que han recurrido a ella son Johnston y Thompson (1989), Laxon, Coltheart y Keating (1988), Rayner (1988) y Seymour (1987).

Una tarea especialmente ideada para determinar la capacidad de la memoria de trabajo impidiendo la intervención de las estrategias de repaso y/o de repetición (rehearsal), es la conocida tarea de Brown-Peterson (Tarea 13; 


\section{3}

Brown, 1958; Peterson y Peterson, 1959). El procedimiento original implica la presentación breve de un conjunto de letras y/o sílabas sin sentido seguida inmediatamente por la realización de alguna actividad distractora por parte del sujeto, durante un tiempo variable. Una vez finalizado el tiempo de demora, el sujeto tiene que recordar los ítems iniciales.

Peterson y Peterson (1959) emplearon como tarea distractora el conteo regresivo de tres en tres o de cuatro en cuatro a partir de un número elegido aleatoriamente (por ejemplo, 304, 301, 298, ...), aunque posteriormente se han empleado otros tipos de tareas distractoras. El objeto central de esta situación es evitar el uso de las estrategias de repaso en la memoria de trabajo para medir la duración del trazo de memoria. Con esta técnica, y manipulando el intervalo de retención ocupado por la tarea distractora, se ha demostrado repetidamente que el recuerdo decae rápidamente en unos pocos segundos.

Una técnica afín para estudiar la codificación fonética es la conocida como vocalización concurrente o simultánea (Tarea 14), utilizada por vez primera por Pintner (1913) y desarrollada mucho después por Levy (1975) y Baddeley (1979), entre otros. Similar a la anterior, consiste en hacer repetir al sujeto una serie de dígitos o de palabras sin relación con la tarea, para comprobar su efecto sobre el recuerdo o comprensión de palabras o frases.

Una interesante aplicación de esta tarea en el ámbito hispano-parlante ha sido realizada por Navalón (1987), quien la empleó para el estudio del papel de la memoria de trabajo en la adquisición lectora en niños de habla castellana (cf. también Ato y Navalón, 1983; Navalón, Ato y Rabadán, 1989; Romero y Sánchez Meca, 1989).

Una tarea ampliamente utilizada para el estudio de la capacidad de la memoria a corto y a largo plazo, así como en aprendizaje verbal, consiste en presentar una lista de ítems y pedir al sujeto, tras un intervalo variable, que informe del mayor número posible de los mismos. Este procedimiento, conocido como tarea de recuerdo libre (Tarea 15), implica la presentación de un número variable de ítems uno a uno (entre 10 y 40). El recuerdo puede ser oral o escrito, y el período de recuerdo suele oscilar entre 30 y 120 segundos, aunque también puede no haber límite de tiempo. El calificativo «libre» hace referencia al hecho de que el orden de evocación de los ítems no interviene en el registro de las respuestas.

Normalmente, la ejecución en esta tarea se plasma gráficamente en la denominada curva de posición serial, en la que se observa claramente que los ítems presentados en primer lugar (efecto de primacía) y los últimos (efecto de recencia) se recuerdan mejor que los intermedios; efectos explicables atendiendo al papel de las dos estructuras de memoria implicadas (a largo y a corto plazo).

Esta tarea permite conocer con más detalle la influencia del significado y también algunos procesos del aprendizaje verbal, tales como las estrategias de organización (agrupación según categorías semánticas o recuerdo categorial) y recuerdo del material verbal. Investigaciones sobre procesos lectores que han recurrido a este paradigma son, por ejemplo, las de Bauer y Emhert (1984), Byrne y Arnold (1981), Navalón, Ato y Rabadán (1989) y Vellutino, Scanlon y Tanzman (1988).

Estrechamente relacionada con ésta, las tareas de amplitud de memoria (Tarea 16) presentan al sujeto una secuencia de ítems de longitud variable -normalmente corta - que deberá recordar en el mismo orden de presentación 


\section{4}

(ésta es la principal diferencia con la tarea de recuerdo libre), alternando secuencias de ensayo y de prueba hasta lograr la evocación completa y ordenada de la lista. El pionero de los métodos de recuerdo serial fue Ebbinghaus (1885), lo que las convierte en las veteranas de las que contemplamos en nuestro ámbito de investigación. Ato y Navalón (1983) y Navalón, Ato y Rabadán (1989) han utilizado esta tarea aplicada a la adquisición lectora castellana.

Este paradigma incluye asimismo lo que se conoce como tarea de memoria para el orden (Tarea 16), mediante la cual se evalúa el recuerdo de una serie de estímulos ordenados espacial (de izquierda a derecha) y temporalmente, contrastándose las diferencias en rendimiento entre grupos extremos de habilidad lectora para cada tipo de orden. Este procedimiento ha sido utilizado por Hulme (1981), Schwartz (1984), y con niños de habla castellana por Rabadán y Sánchez Meca (1989).

La tarea de denominación de dibujos (Tarea 17) consiste básicamente en la presentación de un dibujo que lleva incorporado algún tipo de símbolo verbal (letras, palabras pronunciables o no pronunciables, etc.) solicitando al sujeto que nombre el dibujo lo más rápido posible ignorando los símbolos verbales. En el procedimiento original, ideado por Golinkoff y Rosinski (1976), los símbolos se disponen de tal modo que aparecen sobreimpresionados encima del dibujo, de tal forma que dibujos y símbolos compiten dentro de una misma área retiniana. En otras ocasiones, no aparece esta superposición, sino que los dibujos están situados a la izquierda o a la derecha de los símbolos, lo cual exige una estrategia de exploración por parte del sujeto, e implica componentes lectores en mayor medida.

Puesto que la tarea dirige la atención del niño hacia la denominación de dibujos y no al procesamiento de palabras o de letras, éste supuestamente se realizará de manera automática e involuntaria, estimándose tal procesamiento a partir de la diferencia en la rapidez de denominación de las distintas condiciones. Para paliar el posible efecto que puedan producir los estímulos superpuestos como elemento distractor -aumentando, por tanto, el tiempo de ejecución- se suele añadir una condición con símbolos no verbales.

Hemos incluido esta prueba porque se utiliza para evaluar la lectura automática e involuntaria de letras, palabras pronunciables y no pronunciables. También ha sido empleada para examinar los procesos automáticos de lectura y para realizar estimaciones relativas a la codificación fonológica. Una buena ilustración de las posibilidades de esta prueba para el estudio de la relación entre los procesos mnémicos y los de adquisición lectora puede encontrarse en Katz (1986) y Navalón, Ato y Rabadán (1989).

Recientemente, la investigación básica sobre los procesos implicados en el aprendizaje de la lectura ha dedicado grandes esfuerzos para determinar el papel de la conciencia fonológica en esta habilidad. No cabe duda de que la adquisición de la lectura en una lengua alfabética requiere cierta capacidad metalingüística para concienciarse de que las letras escritas representan segmentos de palabras.

Este campo de estudio ha desarrollado un conjunto de tareas de segmentación fonológica (Tarea 18), cpnsistentes en presentar al sujeto una serie de palabras y pedirle que las divida en sus sílabas componentes, registrándose el silabeo a través de mecanismos diversos. En otras variantes, se solicita del sujeto el deletreo de palabras o la eliminación de determinadas sílabas (inicial, central, final). 
Trabajos recientes de Cossu, Shankweiler, Liberman, Katz y Tola (1988), LeCocq (1986) y Morais, Cluytens y Alegría (1984) han relacionado estas tareas con la habilidad lectora. Una excelente revisión de las posibilidades de esta tarea puede consultarse en Morais, Alegría y Content (1987). Con niños de habla castellana pueden citarse los trabajos de Carrillo, Romero y Sánchez Meca (1989) y Maldonado y Sebastián (1987).

Finalmente, mencionaremos un grupo de procedimientos cuyo objetivo es la contrastación de la eficiencia en la pronunciación o vocalización de una serie de ítems. Admite diversas variantes, pero pueden denominarse tareas de pronunciación y rima de palabras, silabras y no-palabras (Tarea 19), ya que se presentan palabras regulares vs. irregulares, o no-palabras pronunciables vs. no pronunciables. Así, Seymour (1987) utilizó una lista en la cual las palabras variaban en longitud (de 3 a 7 letras), frecuencia de uso, concreción/abstracción del significado, función sintáctica y regularidad grafemafonema. En el mismo estudio empleó también una lista de no-palabras con diversa longitud y distinto grado de homofonía (conforme a una palabra de contraste). Snowling y Frith (1981) compararon la velocidad lectora de buenos y malos lectores según la distorsión que presentasen las palabras de un párrafo, que podían ser fonológicamente similares (igual pronunciación), visualmente similares, o escritas con diversos tipos de letra.

Otros trabajos que han recurrido recientemente a estas tareas son los de Bravo, Bermeosolo y Pinto (1988) con niños chilenos, y Bradley $(1988,1989)$, Coltheart, Laxon y Keating (1988), Coltheart, Laxon, Rickard y Elton (1988), Juel (1988) y Stanovich, Nathan y Zolman (1988), con niños angloparlantes.

\section{CONCLUSIONES FINALES}

En el presente trabajo hemos presentado una taxonomía de las tareas experimentales que están siendo utilizadas para determinar las conexiones existentes entre el rendimiento lector en las primeras etapas de su adquisición y aspectos del procesamiento de la información tales como: (a) el funcionamiento de los procesos cognitivos básicos; (b) el uso adecuado de las estrategias de control; (c) la base del conocimiento contenido en la memoria a largo plazo; (d) la velocidad de acceso al léxico; o (e) la codificación fonológica (Bradley, 1986; Jorm, 1983; Stanovich, 1982; Torgesen, 1985). No era nuestro objetivo ofrecer los resultados obtenidos en estas investigaciones, sino poner al día al lector interesado en los aspectos técnicos y metodológicos que se requieren para el estudio de la adquisición de la lectura desde el enfoque cognitivo.

No cabe duda de que este campo de investigación está contribuyendo a desvelar los factores causales implicados en el desarrollo de esta habilidad. Pero también es cierto que no hay un acuerdo entre los investigadores acerca de los procesos que supuestamente miden las diversas tareas experimentales que aquí se han expuesto. En este sentido, la clasificación de las tareas que hemos presentado en la Tabla 1 debe tomarse, pues, como un intento de sistematización tentativo. Pensamos que es preciso unificar criterios referentes a las características de los sujetos, al control de variables importantes tales como el nivel de inteligencia, y a la evaluación del rendimiento lector, para que los resultados puedan interpretarse de forma coherente. 


\section{Referencias}

Aaron, P. (1987). Developmental dyslexia: Is it different from other forms of reading disability? Annals of Dyslexia, 37, 109-125.

Aaron, P. (1989). Dyslexia and Hyperlexia. Dordrecht, Hollanda: Kluwer.

ARNETT, J. L. y Dilollo, V. (1979). Visual information processing in relation to age and to reading ability. Journal of Experimental Child Psychology, 27, 143-152.

Ato, M. y Navalon, C. (1983). Memoria a corto plazo y habilidad lectora. Revista de Psicología General y Aplicada, 38(6), 1117-1134.

Ato, M.; Sanchez-Meca, J.; Lopez-Pina, J. A.; Velandrino, A. P. y Navalon, C. (1984). Operaciones de codificación de la información en escolares murcianos con deficiencias de lectura: Bases orientativas para el desarrollo de un programa de reeducación. Memoria de un Proyecto de Investigación subvencionado por el Convenio Caja de Ahorros Provincial-Universidad de Murcia.

Averbach, E. y Coriell, A. S. (1961). Short-term memory in vision. Bell System Technical Journal, 40, 309-328.

BADCOCK, D. y LovEGROVE, W. (1981). The effects of contrast, stimulus duration, and spatial frequency on visible persistence in normal and specifically disabled readers. Journal of Experimental Psychology: Human Perception E Performance, 7(3), 495-505.

BADDELEY, A. (1979). Working memory and reading. En P. Kolers, M. Wrolstad y H. Bouma (Eds.), Processing of Visible Language (pp. 355-370). Nueva York: Plenum Press.

Baddeley, A. (1986). Working Memory. Oxford: Clarendon Press.

BAUER, R. H. y EMHERT, J. (1984). Information processing in reading-disabled and nondisabled children. Journal of Experimental Child Pschology, 37, 271-281.

BELLER, H. K. (1970). Parallel and serial stages in matching. Journal of Experimental Psychology, 84, 213-219.

BODER, E. (1971). Developmental dyslexia: Prevailing disgnostic concepts and a new diagnostic approach. En H. J. Miklebust (Ed.), Progress in Learning Disabilities vol. II (pp. 293-321). Nueva York: Grune y Stratton.

BODER, E. (1973). Developmental dyslexia: A diagnostic approach based on three atypical reading-spelling patterns. Developmental Medicine y Child Neurology, 15, 663-687.

Bouma, H. y LeGein, C. P. (1979). Dyslexia as a deficient link between normàl vision and normal speech. IPO Annual Progress Report, 14, 95-100.

Bowers, P. G.; STEFFY, R. y TATE, E. (1988). Comparison of the effects of IQ control methods on memory and naming speed predictors of reading disability. Reading Research Quarterly, 23, 304-319.

BRADLEY, L. (1988). Making connections in learning to read and to spell. Applied Cognitive Psychology, 2, 3-18.

Bradley, L. (1989). Predicting learning disabilities. En J. J. Dumont y H. Nakken (Eds.), Learning Disabilities 2: Cognitive, Social, and Remedial Aspects. Amsterdam: Swets Pub.

Brannan, J. R. y Williams, M. C. (1988). Developmental versus sensory deficit effects on perceptual processing in the reading disabled. Perception y Psychophysics, 44(5), 437-444.

Bravo, L.; Bermeosolo, J. y Pinto, G. (1988). Dislexia fonémica: Decodificación-codificación fonémica y comprensión lectora silenciosa. Infancia y Aprendizaje, 44, 21-34.

BREITMEYER, B. G. y GANZ, L. (1976). Implications of sustained and transient channels for theories of visual pattern masking, saccadic suppression and information processing. Psychological Review, 83, 1-36.

BRown, J. (1958). Some tests of the decay theory of immediate memory. Quarterly Journal of Experimental Psychology, 10, 12-21.

ByrNe, B. y Arnold, L. (1981). Dissociation of the recency effect and immediate span: Evidence from beginning readers. British Journal of Psychology, 72, 371-376.

Carrillo, M. S.; Romero, A. y Sanchez-MecA, J. (noviembre, 1989). Conciencia de la segmentación fonológico-silábica y habilidad lectora en niños de babla castellana. Comunicación presentada al "Primer Symposium Nacional de Metodología de las Ciencias Humanas, Sociales y de la Salud", Salamanca.

Cirrin, F. M. (1984). Lexical search speed in children and adults. Journal of Experimental Child Psychology, 37, 158-175.

Colthear T, M. (1980). Iconic memory and visible persistence. Perception y Psychophysics, 27, 183-228.

Coltheart, V.; Laxon, V. J. y Keating, C. (1988): Effects of word imageability and age of acquisition on children's reading. British Journal of Psychology, 79, 1-12.

Coltheart, V.; Laxon, V. J; Rickard, M. y Elton, C. (1988). Phonological recoding in reading for meaning by adults and children. Journal of Experimental Psychology: Learning, Memory y Cognition, 14, 338-397. 
Cossu, G.; Shankweiler, D.; Liberman, I. Y.; Katz, L. y Tol.a, G. (1988). Awareness of phonological segments and reading ability in italian children. Applied Psycholinguistics, 9, 116.

Dilollo, V.; Hanson, D. y Mcintyre, J. S. (1983). Initial stages of visual information processing in dyslexia. Journal of Experimental Psychology: Human Perception y Performance, 9(6), 923-935.

DUANE, D. (1974). A neurological overview of specific language disability for the nonneurologist. Bulletin of the Orton Society, 24, 5-26.

Ebinghaus, H. (1885). Ubver das Gedächtniss (Trad. inglesa: Memory: A Contribution to Experimental Psychology. Dover, 1964).

Eichelman, W. H. (1970). Familiarity effects in the simultaneous matching task. Journal of Experimental Psychology, 86, 275-282.

Ellis, A. (1985). The cognitive neuropsychology of developmental and acquaired dyslexia: A critical survey. Cognitive Neuropsychology, 2, 169-205.

Ellis, N. C. y Miles, T. R. (1977). Dyslexia as a limitation in the ability to process information. Bulletin of the Orton Society, 27, 72-81.

ERIKSEN, C. W. y COLLINS, J. F. (1967). Some temporal characteristics of visual perception. Journal of Experimental Psychology, 74, 476-484.

Foorman, B. R. y Liberman, D. (1989). Visual and phonological processing of words: A comparison of good and poor readers. Journal of Learning Disabilities, 22(6), 349-355.

Frederiksen, J. R. (1977). Assessment of perceptual, decoding, and lexical skill and their relation to reading proficiency. En A. M. Lesgold, J. W. Pellegrino, S. Fokkema y R. Glaser (Eds.), Cognitive Psychology and Instruction. Nueva York: Plenum Press.

FREDERIKSEN, J. R. (1978). A chronometric study of component skills in reading (Report $\mathbf{n}^{\circ}$ 3757(2)). Boston, MA: Bolt, Baranek y Newman.

FREDERIKSEN, J. R: (1980). Component skills in reading: Measurement of individual differences through chronometric analysis. En R. E. Snow, P. A. Federico y W. E. Montague (Eds.), Aptitude, Learning, and Instruction I: Cognitive Process Analysis of Aptitude (pp. 105-138). Hillsdale, NJ: Erlbaum.

Frederiksen, J. R. (1981). Sources of process interactions in reading. En M. A. Lesgold y C. A. Perfetti (Eds.), Interactive Processes in Reading. Hillsdale, NJ: Erlbaum.

FREDERIKSEN, J. R. (1982). A component theory of reading skills and their interpretations. En R. J. Sternberg (Ed.), Handbook of Human Intelligence vol. 1 (pp. 125-180). Hillsdale, NJ: Erlbaum.

Fredman, G. y Stevenson, J. (1988). Reading processes in specific reading retarded and reading backward 13-years-olds. British Journal of Developmental Psychology, 6, 97-108.

Golinkoff, R. M. y Rosinski, R. R. (1976). Decoding, semantic processing, and language comprehension skill. Child Development, 47, 457-463.

Grosser, G. S. y TrzeciaK, G. M. (1981). Durations of recognition for single letters, with and without visual masking, by dyslexic and normal ręaders. Perceptual E Motor Skills, 23, 991995.

HaBer, R. N. y StANDING, L. (1969). Direct measures of short-term visual storage. Quarterly Joumal of Experimental Psychology, 21, 43-54.

Hall, W. S.; White, T. G. y Guthrie, L. (1986). Skilled reading and language development: Some key issues. En J. Orasanu (ed.), Reading Comprebension: From Research to Practice (pp. 89-111). Hillsdale, NJ: Erlbaum.

Healy, A. F. (1976). Detection errors on the word 'the': Evidence for reading units larger than letters. Journal of Experimental Psychology: Human Perception E Performance, 2, 235-242.

Healy, A. F. (1980). Profreading errors on the word 'the'. New evidences of reading units. Journal of Experimental Psychology: Human Perception y Performance, 6, 45-47.

Hidalgo, M. D. y Sanchez-Meca, J. (noviembre, 1989). Un indicador de la velocidad de codificación visual de letras en el aprendizaje de la lectura. Comunicación presentada al "Primer Symposium Nacional de Metodología de las Ciencias Humanas, Sociales y de la Salud», Salamanca.

Holligan, C. y Johnston, R. S. (1988). The use of phonological information by good and poor readers in memory and reading tasks. Memory $\mathcal{E}$ Cognition, 16, 522-532.

Howell, E. R.; Smith, G. A. y Stanley, G. (1981). Reading disability and visual spatial frequency specific effects. Australian Journal of Psychology, 33, 97-102.

Hulme, C. (1981). Reading Retardation and Multi-Sensory Teaching. Londres: Routledge y Kegan Paul.

Humphreys, G. W. y Evet, L. J. (1985). Are there independent lexical and nonlexical routes in word processing? An evaluation of the dual-route theory of reading. Bebavioral $\mathcal{E}$ Brain Sciences, 8, 689-740.

Hunt, R.; Davidson, J. y Lansman, M. (1981). Individual differences in longterm memory access. Memory \& Cognition, 9, 599-608.

Ingram, T. T. S.; Mason, A. W. y BlackBurn, I. (1970). A retrospective study of 82 children with reading disability. Developments in Medical Child Neurology, 12, 271-281. 
Johnson, D. J. y Myкцebust, H. R. (1967). Learning Disabilities, Educational Principles and Practices. Nueva York: Grune y Straton.

JOHNSTON, R. S. y THOMPSON, G. B. (1989). Is dependence on phonological information in children's reading a product of instructional approach? Journal of Experimental Cbild Psycho$\log y, 48,131-145$.

JORM, A. F. (1983). Specific reading retardation and working memory: A review. British Journal of Psychology, 74, 311-342.

JuEL, C. (1988). Learning to read and write: A longitudinal study of 54 children from first through fourth grades. Journal of Educational Psychology, 80, 437-447.

KATZ, R. B. (1986). Phonological deficiencies in children with reading disability: Evidence from an object-naming task. Cognition, 22, 225-257.

LAXON, V. J.; COLTHEART, V. y KEATING, C. (1988). Children find friendly words friendly too: Words with many orthographic neighbours are easier to read and spell. British Journal of Educational Psychology, 58, 103-119.

LeCoCQ, P. (1986). Sensibilité a la similarité phonétique chez les enfants dyslexiques et les bons lecteurs. L'Anée Psychologique, 86, 201-221.

LesGOLD, A. (1983). A rationale for computer-based reading instruction. En A. C. Wilkinson (Ed.), Classroom Computers and Cognitive Science (pp. 145-163). University of Wisconsin Press.

LesGold, A.; Resnick, L. y Hammond, K. (1985). Learning to read: A longitudinal study of word skill development in two curricula. En E. McKinnon y T. Waller (Eds.), Reading Research: Advances in Theory and Practice vol IV (pp. 107-138). Nueva York: Academic Press.

LeVy, B. A. (1975). Vocalization and suppresion effects in sentence memory. Journal of Verbal Learning \& Verbal Behavior, 14, 304-316.

Lopez-PINA, J. A. (diciembre, 1984). Un nuevo procedimiento experimental para el análisis diferencial de problemas de lectura: Resultados iniciales. Comunicación presentada al «Symposium sobre Actividad Humana y Procesos Cognitivos", Madrid.

LOUBSER, N. y Sharrat, P. (1982). Brief visual memory processes in reading-disabled children. South African Journal of Psychology, 12, 7-18.

Lovegrove, W. y Brown, C. (1978). Development of information processing in normal and disabled readers. Perceptual \& Motor Skills, 46, 1047-1054.

Lóvegrove, W.; Martin, F. y Slaghuis, W. (1986). A theoretical and experimental case of a visual deficit in specific reading disability. Cognitive Neuropsychology, 3, 225-267.

Maldonado, A. y Sebastian, E. (1987). La segmentación de palabras: Un prerrequisito del aprendizaje de la lectura. Boletín del ICE (U.A.M.), 9 (enero), 5-14.

Martin, F. y Lovegrove, W. (1984). The effects of field size and luminance on contrast sensitivity differences between specifically reading disabled and normal children. Neuropsychologia, 22(1), 73-76.

Martin, F. y Lovegrove, W. (1987). Flicker contrast sensitivity in normal and specifically disabled readers. Perception, 16, 215-221.

Morais, J.; Cluytens, M. y Alegria, J. (1984). Segmentation abilities of dyslexics and normal readers. Perceptual \& Motor Skills, 58, 221-222.

Morais, J; Alegria, J. y Content, A. (1987). Segmental analysis and literacy. Cahiers de Psychologie Cognitive, 7(5), 415-556.

MORRISON, F. J.; GIORDANI, B. y NAGY, J. (1977). Reading disability: An information processing analysis. Science, 196, 77-79.

Navalon, C. (1987). El papel de la memoria de trabajo en la adquisición lectora en niños de babla castellana. Tesis Doctoral inédita. Dpto. Metodología y Análisis del Comportamiento, Universidad de Murcia.

Navalon, C.; Ato, M. y Rabadan, N. (1989). El papel de la memoria de trabajo en la adquisición lectora en niños de habla castellana. Infancia y Aprendizaje, 45, 85-106.

NEISSER, U. (1963). Decision-time without reaction-time: Experiments in visual scanning. American Journal of Psychology, 76, 376-385.

Ol.son, R. K.; KLIEGL, R.; Davidson, B. J. y Foltz, G. (1985). Individual and developmental differences in reading disability. En G. E. McKinnon y T. G. Waller (Ed.), Reading Research: Advances in Theory and Practice vol. 4 (pp. 1-64). Nueva York: Academic Press.

Ol.son, R.; Wise, B.; Conners, F.; Rack, J. y Fulker, D. (1989). Specific deficits in component reading and language skills: Genetic and environmental influences. Journal of Learning Disabilities, 22(6), 339-348.

Peterson, L. R. y Peterson, M. J. (1959). Short-term retention of individual items. Journal of Experimental Psychology, 58, 193-198.

PINTNER, R. (1913). Inner speech during silent reading. Psychological Review, 20, 129-153.

Pirozzolo, F. J. (1979). The Neuropsychology of Developmental Reading Disorders. Nueva York: Praeger.

Posner, M. I. y Mitchell, R. F. (1967). Chronometric analysis of classification. Psychological Review, 74, 392-409.

RabADAN, R. y SANCHEz-Meca, J. (noviembre, 1989). La tarea de memoria para el orden como 
un indicador de problemas de lectura. Comunicación presentada al «Primer Symposium Nacional de Metodología de las Ciencias Humanas, Sociales y de la Salud», Salamanca.

RAYNER, K. (1988). Word recognition cues in children: The relative use of graphemic cues, orthografic cues, and grapheme-phoneme correspondence rules. Journal of Educational Psycho$\log y, 80(4), 473-479$.

REICHER, G. M. (1969). Perceptual recognition as a function of meaningfulness of stimulus material. Journal of Experimental Psychology, 81, 274-280.

Resnick, L. B. y WeAver, P. A. (Eds.) (1979). Theory and Practice of Early Reading (3 vols.). Hillsdale, NJ: Erlbaum.

Romero, A. y SANCHEZ-MECA, J. (noviembre, 1989). El papel de la memoria de trabajo en el aprendizaje de la lectura. Comunicación presentada al "Primer Symposium Nacional de Metodología de las Ciencias Humanas, Sociales y de la Salud,, Salamanca.

Schwartz, S. (1984). Measuring Reading Competence: $A$ Theoretical-Prescriptive Approach. Nueva York: Plenum Press.

Seymour, P. H. K. (1986). Cognitive Analysis of Dyslexia. Nueva York: Routledge y Kegan Paul.

SEYMOUR, P. H. K. (1987). Individual cognitive analysis of competent and impaired reading. British Journal of Psychology, 78, 483-506.

SNOWLING, M. y FRITH, U. (1981). The role of sound, shape and orthographic cues in early reading. British Journal of Psychology, 72, 83-87.

SPERLING, G. (1960). The information avalaible in brief visual presentations. Psychological Monographs, 74, 1-29 (traducc. al castellano en M. V. Sebastián (comp.), Lecturas de Psicología de la Memoria. Madrid: Alianza, 1983).

STANLEY, G. (1975). Visual memory processes in dyslexia. En D. Deutsch y A. Deutsch (Eds.), Short-Term Memory (pp. 181-194). Nueva York: Academic Press.

STANLEY, G. y HALL, R. (1973). A comparison of dyslexics and normals in recalling letter arrays after brief presentation. British Journal of Educational Psychology, 43, 301-304.

STANOVICH, K. E. (1982). Individual differences in the cognitive processes of reading: I. Wrod decoding. Journal of Learning Disabilities, 15, 485-493.

STANOVICH, K. E. (1985). Explaining the variance in reading ability in terms of psychological processes: What have we learned? Annals of Dyslexia, 35, 67-96.

STANOVICH, K. E. (1989). Various varying views on variation. Journal of Learning Disabilities, 22(6).

STAnovich, K. E.; Nathan, R. G. y Zolman, J. E. (1988). The developmental lag hypothesis in reading: Longitudinal and matched reading-level comparisons. Child Development, 59, 7186.

Stelmack, R. M.; Saxe, B. J.; Noldy-Cullum, N.; Campbell, K. B. y Armitage, R. (1988). Recognition memory for words and event-related potentials: A comparison of normal and disabled readers. Journal of Clinical y Experimental Neuropsychology, 10, 185-200.

STERnBERG, S. (1969). Mental scanning: Mental processes revealed by reaction time experiments. American Scientist, 57, 421-457.

Szeszulsk1, P. A. y Manis, F. R. (1987). A comparison of word recognition processes in dyslexic and normal readers at two reading-age levels. Journal of Experimental Cbild Psychology, 44, 364-376.

Torgesen, J. K. (1985). Memory processes in reading disabled children. Journal of Learning Disabilities, 18, 350-357.

Tudela, P. (1981). Psicología Experimental. Madrid: UNED.

Tulving (1972). Episodic and semantic memory. En E. Tulving y W. Donaldson (Eds.), Organization of Memory (pp. 361-403). Nueva York: Academic Press.

VAlerA, A. y SANCHEZ-MeCA, J. (noviembre, 1989). La tarea de enmascaramiento visual y su relevancia en el retraso específico en lectura. Comunicación presentada al «Primer Symposium Nacional de Metodología de las Ciencias Humanas, Sociales y de la Salud», Salamanca.

Velandrino, A. P. (1985). La tarea de identificación de anagramas y el proceso de codificación. Anales de Psicología, 2, 129-143.

Velandrino, A. P. y Sanchez-Meca, J. (noviembre, 1989). Acceso al léxico y adquisición lectora. Comunicación presentada al «Primer Symposium Nacional de Metodología de las Ciencias Humanas, Sociales y de la Salud», Salamanca.

Vlillutino, F. R.; SCAnlon, D. M. y Tanzman, M. S. (1988). Lexical memory in poor and normal readers: Developmental differences in the use of category cues. Canadian Journal of Psychology, 42, 216-214.

Williams, M. C. y Lecluyse, K. (En prensa). The perceptual consequences of a temporal processing in reading disabled children. Journal of the American Optometric Association.

WILIIAMs, M. C.; Lecluyse, K. y BologNa, N. (En prensa). Masking by light as a measure of visual integration time in normal and disabled readers. Clinical Vision Sciences.

Williams, M. C.; Molinet, K. y LecluYSE, K. (1989). Visual masking of temporal processing in normal and disabled readers. Clinical Vision Sciences, 4(2), 137-144.

WOLFORD, G. y Fowler, C. A. (1984). Differential use of partial information by good and poor readers. Developmental Review, 4, 16-35. 